\title{
THE SZEGÖ KERNEL ON A CLASS OF NONCOMPACT CR MANIFOLDS OF HIGH CODIMENSION
}

\author{
ANDREW RAICH AND MICHAEL TINKER
}

\begin{abstract}
We generalize Nagel's formula for the Szegö kernel and use it to compute the Szegö kernel on a class of noncompact CR manifolds whose tangent space decomposes into one complex direction and several totally real directions. We also discuss the control metric on these manifolds and relate it to the size of the Szegö kernel.
\end{abstract}

\section{INTRODUCTION}

The goal of this note is to derive a formula for the Szegö kernel for a class of polynomial models that are CR manifolds whose maximal complex tangent space is one (complex) dimensional and totally real tangent space is $n$ (real) dimensional. We also discuss the size of the Szegö kernel in relation to the control metric.

When a CR manifold $M$ has a one (complex) dimensional maximal complex tangent space, then it is standard practice to identify $\bar{\partial}_{b}$ with a vector field $\bar{Z}$ that is antiholomorphic and tangential. The Szegö kernel is then the orthogonal projection $S_{M}$ of $L^{2}(M)$ onto $L^{2}(M) \cap \operatorname{ker} \bar{Z}$. In complex analysis, the Szegö kernel is a fundamental object of study, yet very little is known about the Szegö kernel when the tangent space to $M$ has at least two totally real directions. In the case that $M$ is a quadric submanifold (with no hypothesis on the dimensionality of the maximal complex tangent space), then researchers have computed the partial Fourier transform of the $\square_{b}$-heat kernel, from which the partial Fourier transform of the Szegö kernel can be obtained [BR11, CCM09, CCFI11. This article represents the first time that a formula/estimate for the Szegö kernel has been obtained for any example outside of quadrics when $M$ is not of hypersurface type.

In an interesting twist, we will see in Section 3 that the control metric on $S_{M}$ is finite on the manifold where the Szegö kernel in nonzero. This behavior may provide a clue as to the behavior of the Szegö kernel in higher codimensions when for every point, the span of the antiholomorphic vector fields is a strictly smaller dimension than the the dimension of the tangent space.

Let $p_{1}, \ldots, p_{n}: \mathbb{R} \rightarrow \mathbb{R}$ be a collection of $n$ functions and $P=\left(p_{1}, \ldots, p_{n}\right)$. The functions $p_{j}$ will typically be convex polynomials. Our model $M_{P}$ will be a subset of $\mathbb{C} \times \mathbb{C}^{n}$, and we denote coordinates on $\mathbb{C} \times \mathbb{C}^{n}$ by $(z, w)$ where $z=x+i y \in \mathbb{C}$ and $w=t+i s \in \mathbb{C}^{n}$. Set

$$
\frac{\partial}{\partial \bar{w}}=\left(\frac{\partial}{\partial \bar{w}_{1}}, \ldots, \frac{\partial}{\partial \bar{w}_{n}}\right) .
$$

2010 Mathematics Subject Classification. 32A25,32V20, 32W10, $42 \mathrm{~B} 37$.

Key words and phrases. Szegö kernel, high codimension, control metric, polynomial model.

This work was partially supported by a grant from the Simons Foundation (\#280164 to Andrew Raich).

The $n=2$ case in this paper was part of Tinker's Ph.D. thesis which he completed under Raich's supervision. 
Define

$$
M_{P}=\left\{(z, w) \in \mathbb{C} \times \mathbb{C}^{n}: \operatorname{Im} w=P(x)\right\} .
$$

The maximal complex tangent space is spanned by the vector

$$
\bar{Z}=\frac{\partial}{\partial \bar{z}}-i P^{\prime}(x) \cdot \frac{\partial}{\partial \bar{w}} .
$$

Since the maximal complex tangent space has one dimension, the Szegö kernel on $M$ is the orthogonal projection $S: L^{2}(M) \rightarrow L^{2}(M) \cap \operatorname{ker} \bar{Z}$.

We may identify $M$ with $\mathbb{C} \times \mathbb{R}^{n}$ under the identification

$$
(z, t+i P(x)) \longleftrightarrow(z, t) .
$$

Under this identification, the vector field $2 \bar{Z}$ pushes forward to the vector field

$$
\bar{L}=\frac{\partial}{\partial x}+i\left(\frac{\partial}{\partial y}-P^{\prime}(x) \cdot \frac{\partial}{\partial t}\right)
$$

We have a choice of measure to put on $M$ (and consequently on $\mathbb{C} \times \mathbb{R}^{n}$ ). If $n=1$ and $P(x)=x^{2}$, then $M$ is the Heisenberg group $\mathbb{H}^{1}$ and Haar measure on $M$ corresponds to Lebesgue measure on $\mathbb{C} \times \mathbb{R}$. Following precedent [Nag86, Chr91, Rai06b, Rai06a, Rai07, Rai12, BR13b, BR11, BR09, NS06, NRSW89, Has94, [Str09], we use Lebesgue measure on $\mathbb{C} \times \mathbb{R}^{n}$.

We can then identify the Szegö projection $S_{P}$ on $L^{2}(M)$ with a projection that (by an abuse of notation) we also call the Szegö projection and denote by $S_{P}$; namely, the orthogonal projection of $L^{2}\left(\mathbb{C} \times \mathbb{R}^{n}\right)$ onto $L^{2}\left(\mathbb{C} \times \mathbb{R}^{n}\right) \cap$ ker $\bar{L}$. By standard Hilbert space theory, this Szegö projection $S_{P}$ is given by integration against a kernel $S_{P}\left((x, y, t),\left(x^{\prime}, y^{\prime}, t^{\prime}\right)\right)$, that is,

$$
S_{P} f(x, y, t)=\int_{\mathbb{C} \times \mathbb{R}^{n}} S_{P}\left((x, y, t),\left(x^{\prime}, y^{\prime}, t^{\prime}\right)\right) f\left(x^{\prime}, y^{\prime}, t^{\prime}\right) d x^{\prime} d y^{\prime} d t^{\prime} .
$$

The first goal of this paper is to find a tractable expression for $S_{P}\left((x, y, t),\left(x^{\prime}, y^{\prime}, t^{\prime}\right)\right)$.

Theorem 1.1. Let $M_{P}$ be a polynomial model defined by (11). Then the Szegö kernel for $M_{P}$ is given by the formula

$$
S_{P}\left((x, y, t),\left(x^{\prime}, y^{\prime}, t^{\prime}\right)\right)=\int_{\Sigma_{P}} \frac{1}{C_{\eta, \tau}} e^{2 \pi \eta\left(\left(x+x^{\prime}\right)+i\left(y-y^{\prime}\right)\right)} e^{-2 \pi \tau \cdot\left(P(x)+P\left(x^{\prime}\right)-i\left(t-t^{\prime}\right)\right)} d \eta d \tau
$$

where

$$
C_{\eta, \tau}=\int_{\mathbb{R}} e^{4 \pi\left(x^{\prime} \eta-P\left(x^{\prime}\right) \cdot \tau\right)} d x^{\prime}
$$

and $\Sigma_{P}=\left\{(\eta, \tau) \in \mathbb{R} \times \mathbb{R}^{n}: C_{\eta, \tau}<\infty\right\}$.

Theorem 1.1 generalizes the Szegö kernel formula of Nagel [Nag86, p.302]. In [Nag86, Nagel investigates the case $M_{p}=\left\{(z, w) \in \mathbb{C}^{2}: \operatorname{Im} w=p(w)\right\}$ where $p$ is a convex polynomial. If $C_{\eta, r}^{1}=\int_{\mathbb{R}} e^{4 \pi(\eta x-r p(x))} d x$ and $\Sigma_{p}=\left\{(\eta, r): C_{\eta, r}^{1}<\infty\right\}$, then Nagel proves that

$$
S_{p}\left((x, y, t),\left(x^{\prime}, y^{\prime}, t^{\prime}\right)\right)=\int_{\Sigma_{p}} \frac{1}{C_{\eta, \tau}^{1}} e^{2 \pi \eta\left(\left(x+x^{\prime}\right)+i\left(y-y^{\prime}\right)\right)} e^{-2 \pi \tau \cdot\left(p(x)+p\left(x^{\prime}\right)-i\left(t-t^{\prime}\right)\right)} d \eta d \tau .
$$

We now explore several consequences of Theorem 1.1. 
Theorem 1.2. Let $M_{P}$ be a model defined by (11), and assume that $p_{j}(x)=a_{j} p(x)$ for $1 \leq j \leq n$ where $a_{n}=1$ and $p(x)$ is a smooth function satisfying $\lim _{|x| \rightarrow \infty} \frac{p(x)}{|x|}=\infty$. If we let $t=\left(s, t_{n}\right)$ and $a=(b, 1)$, then

$$
S_{P}\left((x, y, t),\left(x^{\prime}, y^{\prime}, t^{\prime}\right)\right)=\delta_{0}\left[\left(s-s^{\prime}\right)-b\left(t_{n}-t_{n}^{\prime}\right)\right] S_{p}\left(\left(x, y, t_{n}\right),\left(x^{\prime}, y^{\prime}, t_{n}^{\prime}\right)\right)
$$

where $\delta_{0}$ is the Dirac- $\delta$ in $\mathbb{R}^{n-1}$.

The size of the Szegö kernel when $M_{p}$ is a model of three real dimensions and $p$ is a convex polynomial is well understood Nag86, NRSW89, Chr91, Rai06a. In particular if $d(\cdot, \cdot)$ is the control metric generated by the vector fields $X_{1}=\operatorname{Re} \bar{L}$ and $X_{2}=\operatorname{Im} \bar{L}$, and $B_{C C}(\alpha, \delta)$ is the control ball of radius $\delta$, then if $X^{J}$ is a multiindex of operators $X_{1}, X_{2}$ acting in either $\alpha=(x, y, t)$ or $\beta=\left(x^{\prime}, y^{\prime}, t^{\prime}\right)$, then $\left|X^{J} S_{P}(\alpha, \beta)\right| \lesssim|d(\alpha, \beta)|^{-|J|}\left|B_{C C}(\alpha, d(\alpha, \beta))\right|^{-1}$. This yields an immediate corollary.

Corollary 1.3. Let $M_{P}$ be a model as in Theorem 1.2 where $p$ is a convex polynomial. If $X^{J}$ is a multiindex of operators $X_{1}, X_{2}$ acting in either $\alpha=(x, y, t)$ or $\beta=\left(x^{\prime}, y^{\prime}, t^{\prime}\right)$, then there exists a constant $C_{|J|}>0$ so that on $\operatorname{supp} \delta_{0}\left[\left(s-s^{\prime}\right)-b\left(t_{n}-t_{n}^{\prime}\right)\right]$

$$
\left|X^{J} S_{M}(\alpha, \beta)\right| \leq C_{|J|} \frac{|d(\alpha, \beta)|^{-|J|}}{\left|B_{C C}(\alpha, d(\alpha, \beta))\right|} .
$$

The proof is immediate, given the fact that $X_{1}$ and $X_{2}$ are tangential on the manifold where $s-s^{\prime}=b\left(t_{n}-t_{n}^{\prime}\right)$.

If $p_{j}(x)=a_{j} x^{2}$, then $M$ is an example of quadric submanifold. Quadrics have been studied extensively [BR11, BR13a, CT00, CCT06, BGG96, BGG00, Gav77, Hul76] and [CCM09], in particular for a more extensive background. In this case, we can compute all of the integrals explicitly and prove the following theorem.

Theorem 1.4. Let $M_{P}$ be the quadric submanifold defined by

$$
M_{a|x|^{2}}=\left\{(z, w) \in \mathbb{C} \times \mathbb{C}^{n}: \operatorname{Im} w=x^{2} a\right\}
$$

where $a=\left(a_{1}, \ldots, a_{n}\right) \in \mathbb{R}^{n}$ and $a_{n}>0$. Then

$$
\begin{aligned}
S_{a|x|^{2}}\left((x, y, t),\left(x^{\prime}, y^{\prime}, t^{\prime}\right)\right) & =S_{a_{n}|x|^{2}}\left(\left(x, y, t_{n}\right),\left(x^{\prime}, y^{\prime}, t_{n}^{\prime}\right)\right) \delta_{0}\left[a_{n}\left(s-s^{\prime}\right)-b\left(t_{n}-t_{n}^{\prime}\right)\right] \\
& =\frac{2 a_{n} \delta_{0}\left[a_{n}\left(s-s^{\prime}\right)-b\left(t_{n}-t_{n}^{\prime}\right)\right]}{\left(\pi a_{n}\left[\left(x-x^{\prime}\right)^{2}+\left(y-y^{\prime}\right)^{2}\right]-2 \pi i\left[\left(t_{n}-t_{n}^{\prime}\right)+a_{n}\left(x+x^{\prime}\right)\left(y-y^{\prime}\right)\right]\right)^{2}}
\end{aligned}
$$

Remark 1.5. The condition that $a_{n}>0$ is not essential - we really require that $a_{n} \neq 0$, but we keep $a_{n}>0$ for simplicity. Also, in the proof of Theorem 1.4 we explicitly compute the $S_{\lambda x^{2}}\left((x, y, t),\left(x^{\prime}, y^{\prime}, t^{\prime}\right)\right)$ where $\lambda>0$, and from that expression, we can see there is nothing distinguished about the $n$th coordinate, except the fact that $a_{n} \neq 0$.

The outline of the remainder of the paper consists of the proofs of the main theorems in Section 2 and a discussion of the control geometry in Section 3 .

\section{Proofs of the Main Theorems}

\subsection{Proof of the Szegö kernel formula.}


Proof of Theorem 1.1. The proof of Theorem 1.1 follows from two observations. The first is that $\bar{L}$ is translation invariant in $y$ and $t$. This means we can take the partial Fourier transform in $y$ and $t$. Given a function $f(x, y, t)$, we define the partial Fourier transform of $f$ to be

$$
\mathcal{F} f(x, \eta, \tau)=\hat{f}(x, \eta, \tau)=\iint_{\mathbb{R} \times \mathbb{R}^{n}} e^{-2 \pi i(y, t) \cdot(\eta, \tau)} f(x, y, t) d y d t .
$$

Under $\mathcal{F}$, with $(\eta, \tau)$ as the transform variables of $(y, t)$, the operator

$$
\bar{L} \mapsto \hat{\bar{L}}=\frac{\partial}{\partial x}-2 \pi \eta+2 \pi P^{\prime}(x) \cdot \tau=e^{2 \pi(x \eta-P(x) \cdot \tau)} \frac{\partial}{\partial x} e^{-2 \pi(x \eta-P(x) \cdot \tau)} .
$$

Set $\Psi(x, \eta, \tau)=e^{-2 \pi(x \eta-P(x) \cdot \tau)}$ and $M_{\Psi}: L^{2}(\mathbb{R}, d x) \rightarrow L^{2}\left(\mathbb{R}, e^{4 \pi(x \eta-P(x) \cdot \tau)}\right)$ to be the isometry defined by $f \mapsto \Psi f$. Since $M_{\Psi}$ and $\mathcal{F}$ are isometries, $\bar{L} f=0$ if and only if $\frac{d}{d x}\left\{M_{\Psi} \mathcal{F} f\right\}=0$.

The second observation is that ker $\frac{d}{d x}$ are constant functions. The function $f=1$ is in $L^{2}\left(\mathbb{R}, e^{4 \pi(x \eta-P(x) \cdot \tau)}\right)$ exactly when $C_{\eta, \tau}<\infty$. Assuming $C_{\eta, \tau}<\infty$, then the projection of $g$ onto ker $\frac{d}{d x}$ is the operator $P_{\eta, \tau}$ given by

$$
P_{\eta, \tau} g(x)=P_{\eta, \tau} g=\frac{1}{C_{\eta, \tau}} \int_{\mathbb{R}} g\left(x^{\prime}\right) e^{4 \pi\left(x^{\prime} \eta-P(x) \cdot \tau\right)} d x^{\prime} .
$$

If the operator $P=P_{\eta, \tau}$ on $L^{2}\left(\mathbb{R}, e^{4 \pi(x \eta-P(x) \cdot \tau)}\right)$ with the understanding that $P_{\eta, \tau}=0$ when $(\eta, \tau) \notin \Sigma$. Consequently,

$$
S=\mathcal{F}^{-1} M_{\Psi^{-1}} P M_{\Psi} \mathcal{F} .
$$

Expanding the right-hand side yields the desired formula.

\subsection{Proof of the Szegö kernel formula when $P=a p$.}

Proof of Theorem 1.2. We use the following notation: $a=\left(a_{1}, \ldots, a_{n}\right), b=\left(a_{1}, \ldots, a_{n-1}\right)$, $\tau=\left(\sigma, \tau_{n}\right)$. Also, $a \cdot \tau=\tau_{n}+b \cdot \sigma$. Since $\lim _{|x| \rightarrow \infty} \frac{p(x)}{|x|}=\infty, C_{\eta, \tau}<\infty$ if and only if $a \cdot \tau>0$ (which is equivalent to $\tau_{n}>-b \cdot \sigma$ ), and this condition is independent of $\eta$. We use a superscript to denote which model to which various expressions refer. Also, $P(x)=p(x) a$, So

$$
C_{\eta, \tau}^{a p}=\int_{\mathbb{R}} e^{4 \pi(x \eta-p(x) a \cdot \tau)} d x=C_{\eta, \tau_{n}+\sigma \cdot b}^{p} .
$$

Consequently, we use Theorem 1.1 and compute that

$$
\begin{aligned}
& S_{a p}\left((x, y, t),\left(x^{\prime}, y^{\prime}, t^{\prime}\right)\right)=\int_{\Sigma_{a p}} \frac{1}{C_{\eta, \tau}^{a p}} e^{2 \pi \eta\left(x+x^{\prime}+i\left(y-y^{\prime}\right)\right)} e^{-2 \pi \tau \cdot\left(a\left(p(x)+p\left(x^{\prime}\right)\right)-i\left(t-t^{\prime}\right)\right)} d \tau d \eta \\
& =\int_{\mathbb{R}^{n-1}} \int_{\mathbb{R}} \int_{\tau_{n}=-b \cdot \sigma}^{\infty} \frac{1}{C_{\eta, \tau_{n}+\sigma \cdot b}^{p}} e^{2 \pi \eta\left(x+x^{\prime}+i\left(y-y^{\prime}\right)\right)} e^{-2 \pi\left(\tau_{n}+b \cdot \sigma\right)\left(p(x)+p\left(x^{\prime}\right)-i\left(t_{n}-t_{n}^{\prime}\right)\right)} e^{2 \pi i \sigma \cdot\left[\left(s-s^{\prime}\right)-b\left(t_{n}-t_{n}^{\prime}\right)\right]} d \tau_{n} d \eta d \sigma
\end{aligned}
$$

where the last line uses the equality $\tau \cdot\left(t-t^{\prime}\right)=\sigma \cdot\left(s-s^{\prime}\right)+\tau_{n}\left(t_{n}-t_{n}^{\prime}\right)$ and the fact that $a_{n}=1$. Shifting the variable $\tau+b \cdot \sigma \mapsto \tau$, comparing the resulting formula to (2), and recognizing that resulting integration in $\sigma$ results in a $\delta_{0}\left[\left(s-s^{\prime}\right)-b\left(t_{n}-t_{n}^{\prime}\right)\right]$ finishes the proof. 


\subsection{The quadric case.}

Proof of Theorem 1.4. We use Theorem 1.2. In the case that $p(x)=x^{2}$ and $n=1$, we see that if $\lambda>0$, then $C_{\eta, \tau}^{\lambda x^{2}}=\frac{e^{\frac{\pi \eta^{2}}{\lambda \tau}}}{2 \sqrt{\lambda \tau}}$, so applying Nagel's formula yields

$$
\begin{aligned}
S_{\lambda x^{2}}\left((x, y, t),\left(x^{\prime}, y^{\prime}, t^{\prime}\right)\right) & =\int_{0}^{\infty} \int_{\mathbb{R}} 2 \sqrt{\tau \lambda} e^{-\frac{\pi \eta^{2}}{\lambda \tau}} e^{-2 \pi \tau\left[\lambda\left(x^{2}+x^{\prime 2}\right)-i\left(t-t^{\prime}\right)\right]} e^{2 \pi \eta\left[\left(x+x^{\prime}\right)+i\left(y-y^{\prime}\right)\right]} d \eta d \tau \\
& =\frac{2 \lambda}{\left(\pi \lambda\left[\left(x-x^{\prime}\right)^{2}+\left(y-y^{\prime}\right)^{2}\right]-2 \pi i\left[\left(t-t^{\prime}\right)+\lambda\left(x+x^{\prime}\right)\left(y-y^{\prime}\right)\right]\right)^{2}}
\end{aligned}
$$

We are not assuming that $a_{n}=1$, and we could use a change of variables to reduce to this case, but it is simpler to make the change of variables $\tilde{\sigma}=\sigma$, and $\tilde{\tau}_{n}=a \cdot \tau$. Next, an easy

computation establishes that $\Sigma_{a x^{2}}=\{(\eta, \tau): a \cdot \tau>0\}=\left\{(\eta, \tilde{\tau}): \tilde{\tau}_{n}>0\right\}$ and $C_{\eta, \tau}^{a x^{2}}=\frac{e^{\frac{\pi \eta^{2}}{a \cdot \tau}}}{2 \sqrt{a \cdot \tau}}$ Consequently, we compute that $\tau_{n}=\left(\tilde{\tau}_{n}-\sigma \cdot b\right) / a_{n}$ and

$$
\begin{aligned}
& S_{a x^{2}}\left((x, y, t),\left(x^{\prime}, y^{\prime}, t^{\prime}\right)\right)=\int_{\Sigma_{a x^{2}}} 2 \sqrt{a \cdot \tau} e^{-\frac{\pi \eta^{2}}{a \cdot \tau}} e^{2 \pi \eta\left[\left(x+x^{\prime}\right)+i\left(y-y^{\prime}\right)\right]} e^{-2 \pi \tau \cdot\left[a\left(x^{2}+x^{\prime 2}\right)-i\left(t-t^{\prime}\right)\right]} d \eta d \tau \\
& =\int_{\mathbb{R}} \int_{0}^{\infty} \int_{\mathbb{R}^{n-1}} 2 \sqrt{\tilde{\tau}_{n}} e^{-\frac{\pi \eta^{2}}{\tilde{\tau}_{n}}} e^{2 \pi \eta\left[\left(x+x^{\prime}\right)+i\left(y-y^{\prime}\right)\right]} e^{-2 \pi \tilde{\tau}_{n}\left(x^{2}+x^{\prime 2}\right)} e^{2 \pi i \sigma \cdot\left(s-s^{\prime}\right)} e^{2 \pi i\left(\tilde{\tau}_{n}-\sigma \cdot b\right)\left(t_{n}-t_{n}^{\prime}\right) / a_{n}} d \sigma d \tilde{\tau}_{n} d \eta \\
& =\frac{1}{a_{n}} \int_{\mathbb{R}} \int_{0}^{\infty} \int_{\mathbb{R}^{n-1}} 2 \sqrt{\tilde{\tau}_{n}} e^{-\frac{\pi \eta^{2}}{\tilde{\tau}_{n}}} e^{2 \pi \eta\left[\left(x+x^{\prime}\right)+i\left(y-y^{\prime}\right)\right]} e^{-2 \pi \tilde{\tau}_{n}\left[\left(x^{2}+x^{\prime 2}\right)-i\left(t_{n}-t_{n}^{\prime}\right) / a_{n}\right]} e^{2 \pi i \sigma \cdot\left[\left(s-s^{\prime}\right)-\frac{b}{a_{n}}\left(t_{n}-t_{n}^{\prime}\right)\right]} d \sigma d \eta d \tilde{\tau}_{n} \\
& =\frac{1}{a_{n}} \frac{2 \delta_{0}\left[a_{n}\left(s-s^{\prime}\right)-b\left(t_{n}-t_{n}^{\prime}\right)\right]}{\left(\pi\left[\left(x-x^{\prime}\right)^{2}+\left(y-y^{\prime}\right)^{2}\right]+2 \pi i\left[\left(t_{n}-t_{n}^{\prime}\right) / a_{n}+\left(x+x^{\prime}\right)\left(y-y^{\prime}\right)\right]\right)^{2}} \\
& =S_{a_{n}|x|^{2}}\left(\left(x, y, t_{n}\right),\left(x^{\prime}, y^{\prime}, t_{n}^{\prime}\right)\right) \delta_{0}\left[a_{n}\left(s-s^{\prime}\right)-b\left(t_{n}-t_{n}^{\prime}\right)\right] .
\end{aligned}
$$

\section{Connection to the Control Geometry}

Since on a finite type domain boundary in $\mathbb{C}^{2}$ the Szegö kernel is governed by the control metric, we may naturally ask whether the same holds on a codimension CR manifold with at least two totally real directions. It is easy to extend the notion of finite commutator type; we simply require that the real and imaginary parts of $\bar{L}, X_{1}$ and $X_{2}$, along with a finite number $m$ of their iterated commutators, span the real tangent space at every point of $M_{P}$.

Definition 3.1. With notation as above, let $\left\{Y_{1}, \ldots, Y_{q}\right\}$ be some enumeration of the vector fields $X_{1}, X_{2}$, and all their iterated commutators of length less than or equal $m$. Define the "degree" of each vector field $Y_{j}$ by

$$
\mathrm{d}\left(Y_{j}\right)=\text { length of the iterated commutator that forms } Y_{j}
$$

Now let the distance between $p, q \in M_{P}$ be the infimum of $\delta>0$ such that there is an absolutely continuous map $\gamma:[0,1] \mapsto M_{P}$ with $\gamma(0)=p, \gamma(1)=q$ so that for almost all $r \in(0,1)$

$$
\gamma^{\prime}(r)=\sum_{j=1}^{q} c_{j}(t) Y_{j}(\gamma(r)), \quad\left|c_{j}(r)\right|<\delta^{\mathrm{d}\left(Y_{j}\right)}
$$


Under this condition, the control distance yields a metric, but one which currently defies any tractable description. (Indeed, even on a domain boundary $M_{p}$, a serious amount of work is required to prove the equivalence of the control metric to the pseudometrics investigated by Nagel et al. NSW85.) Although $M_{a p(x)}$ is not of finite type, there is a submanifold of $M_{a p(x)}$ on which the control distance is finite and a direct connection to the Szegö kernel on $M_{a p(x)}$. Here $X_{1}=\frac{\partial}{\partial x}$ and $X_{2}=\frac{\partial}{\partial y}-p^{\prime}(x) a \cdot \frac{\partial}{\partial t}$, so every potentially non-zero commutator is of the form

$$
\left.\left.Y_{k}=[\overbrace{X_{1},\left[X_{1}, \cdots,\left[X_{1}\right.\right.}^{k-1}, X_{2}] \cdots\right]\right] \quad(2 \leq k \leq m)
$$

That is,

$$
Y_{k}=-p^{(k)}(x) a \cdot \frac{\partial}{\partial t} .
$$

This forces $\left\{X_{1}, X_{2}, Y_{k_{1}}, Y_{k_{2}}\right\}$ to span only the subspace generated by $\left\{\frac{\partial}{\partial x}, \frac{\partial}{\partial y}, a \cdot \frac{\partial}{\partial t}\right\}$. Thus the real tangent space is never spanned, at any point of $M_{a p(x)}$, and $\left\{X_{1}, X_{2}\right\}$ do not generate a finite control metric.

We may still consider control distance on $M_{a p(x)}$. This distance is finite and less than some $\delta>0$ if and only if there exists an absolutely continuous curve $\gamma:[0,1] \mapsto M_{a p(x)}$ such that

$$
\gamma^{\prime}(\varsigma)=c_{0}(\varsigma) X_{1}(\gamma(\varsigma))+c_{1}(\varsigma) X_{2}(\gamma(\varsigma))+\sum_{k=2}^{m} c_{k}(\varsigma) Y_{k}(\gamma(\varsigma))
$$

with $\left|c_{0}(\varsigma)\right|,\left|c_{1}(\varsigma)\right|<\delta$ and $\left|a_{k}(\varsigma)\right|<\delta^{k}$ for almost all $\varsigma \in(0,1)$. Given our previous comments, such a curve $\gamma(\varsigma)=\left(\gamma_{1}(\varsigma), \gamma_{2}(\varsigma), \gamma_{3}(\varsigma), \ldots, \gamma_{n+2}(\varsigma)\right)$ can only exist if $\left(\gamma_{3}^{\prime}, \ldots, \gamma_{n+2}^{\prime}\right)$ is parallel to $a$ for almost all $\varsigma \in(0,1)$. From Theorem 1.2, the Szegö kernel on $M_{a p(x)}$ is a singular distribution supported on exactly the subspace where the control distance on $M_{a p(x)}$ is finite. On this subspace, the control ball is well-defined and exactly determines the size of the Szegö kernel on $M_{a p(x)}$, treating the subspace as an $\mathbb{R}^{3}$ and applying the Nagel et. al. machinery.

\section{REFERENCES}

[BGG96] R. Beals, B. Gaveau, and P.C. Greiner. The Green function of model step two hypoelliptic operators and the analysis of certain tangential Cauchy Riemann complexes. Adv. Math., 121(2):288$345,1996$.

[BGG00] R. Beals, B. Gaveau, and P.C̃. Greiner. Hamilton-Jacobi theory and the heat kernel on Heisenberg groups. J. Math. Pures Appl. (9), 79(7):633-689, 2000.

[BR09] A. Boggess and A. Raich. A simplified calculation for the fundamental solution to the heat equation on the Heisenberg group. Proc. Amer. Math. Soc., 137(3):937-944, 2009.

[BR11] A. Boggess and A. Raich. The $\square_{b}$-heat equation on quadric manifolds. J. Geom. Anal., 21:256$275,2011$.

[BR13a] A. Boggess and A. Raich. Fundamental solutions to $\square_{b}$ on certain quadrics. J. Geom. Anal., 23(4):1729-1752, 2013.

[BR13b] A. Boggess and A. Raich. Heat kernels, smoothness estimates and exponential decay. J. Fourier Anal. Appl., 19:180-224, 2013.

[CCFI11] O. Calin, D.-C. Chang, K. Furutani, and C. Iwasaki. Heat kernels for elliptic and sub-elliptic operators. Applied and Numerical Harmonic Analysis. Birkhäuser/Springer, New York, 2011.

[CCM09] O. Calin, D.-C. Chang, and I. Markina. Generalized Hamilton-Jacobi equation and heat kernel on step two nilpotent Lie groups. In Analysis and mathematical physics, Trends Math., pages 49-76. Birkhäuser, Basel, 2009. 
[CCT06] O. Calin, D.-C. Chang, and J. Tie. Fundamental solutions for Hermite and subelliptic operators. J. Anal. Math., 100:223-248, 2006.

[Chr91] M. Christ. On the $\bar{\partial}$ equation in weighted $L^{2}$ norms in $\mathbb{C}^{1}$. J. Geom. Anal., 1(3):193-230, 1991.

[CT00] D.C. Chang and J. Tie. Estimates for powers of the sub-Laplacian on the non-isotropic Heisenberg group. J. Geom. Anal., 10:653-678, 2000.

[Gav77] B. Gaveau. Principe de moindre action, propogation de la chaleur, et estimées sous elliptiques sur certains groupes nilpotents. Acta Math., 139:95-153, 1977.

[Has94] F. Haslinger. Szegö kernels for certain unbounded domains in $\mathbb{C}^{2}$. Travaux de la Conférence Internationale d'Analyse Complexe et du 7e Séminaire Roumano-Finlandais (1993). Rev. Roumaine Math. Pures Appl., 39:939-950, 1994.

[Hul76] A. Hulanicki. The distribution of energy in the Brownian motion in the Gaussian field and analytic hypoellipticity of certain subelliptic operators on the Heisenberg group. Studia Math., 56:165-173, 1976.

[Nag86] A. Nagel. Vector fields and nonisotropic metrics. In Beijing Lectures in Harmonic Analysis, Ann. of Math. Stud., pages 241-306. Princeton University Press, 1986.

[NRSW89] A. Nagel, J.-P. Rosay, E.M. Stein, and S. Wainger. Estimates for the Bergman and Szegö kernels in $\mathbb{C}^{2}$. Ann. of Math., 129:113-149, 1989.

[NS06] A. Nagel and E.M. Stein. The $\bar{\partial}_{b}$-complex on decoupled domains in $\mathbb{C}^{n}, n \geq 3$. Ann. of Math., 164:649-713, 2006.

[NSW85] A. Nagel, E.M. Stein, and S. Wainger. Balls and metrics defined by vector fields I: Basic properties. Acta Math., 155:103-147, 1985.

[Rai06a] A. Raich. Heat equations in $\mathbb{R} \times \mathbb{C}$. J. Funct. Anal., 240(1):1-35, 2006.

[Rai06b] A. Raich. One-parameter families of operators in C. J. Geom. Anal., 16(2):353-374, 2006.

[Rai07] A. Raich. Pointwise estimates of relative fundamental solutions for heat equations in $\mathbb{R} \times \mathbb{C}$. Math. Z., 256:193-220, 2007.

[Rai12] A. Raich. Heat equations and the weighted $\bar{\partial}$-problem. Commun. Pure Appl. Anal., 11(3):885909, 2012.

[Str09] B. Street. The $\square_{b}$-heat equation and multipliers via the wave equation. Math. Z., 263(4):861-886, 2009 .

Department of Mathematical Sciences, SCEN 301, 1 University of Arkansas, FayetTEVILLE, AR 72701

E-mail address: araich@uark.edu

Plano, TX

E-mail address: michael.tinker@ca.com 